\title{
DETERMINAÇÃO DAS CONCENTRAÇÕES SÉRICAS DE GLICOSE E INSULINA DE CÃES EM CHOQUE ENDOTÓXICO
}

\author{
EVALUATION OF GLUCOSE AND INSULIN SERUM CONCENTRATIONS OF DOGS IN \\ ENDOTOXIC SHOCK
}

\author{
Márcia Mery Kogika ${ }^{1}$ Leonardo Pinto Brandão ${ }^{2}$ Márcia Marques Jericó ${ }^{3}$ \\ Mitika Kuribayashi Hagiwara ${ }^{4}$ Denise Maria Nunes Simões ${ }^{5}$ \\ Berenice Mendonça ${ }^{6}$
}

\section{RESUMO}

A progressiva instalação de um quadro hipoglicêmico é uma das principais causas de evolução fatal dos animais em choque endotóxico. A terapia preconizada pela literatura sugere a reposição de glicose e insulina a estes pacientes com o objetivo de facilitar a entrada de glicose nos tecidos; no entanto, ha controvérsias com relação aos valores séricos de insulina nos animais em choque endotóxico, gerando dúvidas quanto aos benefícios da insulinoterapia nestes pacientes. O presente estudo determinou as variações nas concentrações séricas de glicose em cães em choque endotóxico e avaliou as concentrações séricas de insulina, em valores absolutos e relativos (relação insulina/glicose e insulina/glicose corrigida), nos animais hipoglicêmicos, obtendo-se subsídios para a instituição de terapia de reposição de insulina e glicose na fase hipodinâmica do choque. Foram estudados 25 cães atendidos no Serviço de Pronto Atendimento Médico do Hospital Veterinário da FMVZ/USP, apresentando quadro de choque endotóxico decorrente de gastrenterite hemorrágica, com diagnóstico presuntivo de parvovirose. Em 52\% dos animais observou-se hipoglicemia; $36 \%$ dos animais estavam normoglicêmicos e $12 \%$ hiperglicêmicos. Dentre os 13 animais hipoglicêmicos observou-se a ocorrência de hiperinsulinemia absoluta e relativa em 3 cães e apenas relativa em 6 animais. Em 2 animais observou-se hipoinsulinemia absoluta. A constatação de que mais da metade dos animais hipoglicêmicos, na fase mais avançada do choque, apresentaram hiperinsulinemia absoluta ou relativa revelou a necessidade de uma avaliação cautelosa do paciente antes de se proceder à indicação terapêutica de insulina, a qual se restringirá àqueles em que houver resistência periférica à sua ação ou ainda redução de sua concentração sérica.
Palavras-chave: choque séptico, cães, insulina, glicose.

\section{SUMMARY}

Progressive hypoglycemia is one of the main causes that could lead dogs to death with septic shock. The ancillary therapy suggested by many authors includes glucose and insulin replacement to improve tissue glucose utilization; however, there are some contradictions about insulin serum concentration values in patients with septic shock, making questionable the need for insulin replacement. This study evaluated glucose serum concentration changes in dogs with septic shock and insulin serum concentrations (absolute and relative ratios) in the hypoglycemic group obtaining information that may help the therapy of hypodinamic shock. Twenty-five dogs seen in the emergency room of the Veterinary Teaching Hospital of Universidade de São Paulo showing signs of endotoxic shock due to a hemorragic gastroenteritis (presumptive diagnosis of parvovirus infection) were evaluated in this study. There were 52\% hypoglycemic animals, $36 \%$ normoglycemic and $12 \%$ were hyperglycemic. Out of 13 hypoglycemic dogs, 3 had absolute and relative hyperinsulinemia and only relative hyperinsulinemia in 6 dogs. Absolute hypoinsulinemia was observed in 2 animals. The finding that more than a half of the animals in the hypodinamic state of shock presented absolute or relative hyperinsulinemia, denoted the demand of carefull laboratorial evaluation of the patient prior to insulin administration. The administration should be reserved for animals with periferic resistance or decrease in insulin serum concentration.

Key words: septic shock, dogs, insulin, glucose.

\footnotetext{
${ }^{1}$ Professor Doutor, Departamento de Clínica Médica, Faculdade de Medicina Veterinária e Zootecnia (FMVZ), Universidade de São Paulo (USP).

${ }^{2}$ Ex-bolsista de iniciação científica PIBIC-CNPq, pós-graduando do Departamento de Clínica Médica, FMVZ, USP. Av. São Remo 463, Apt. 71-A, B1-2. Ed. Guadalupe - Vila Universitária, 05360-150. E-mail: leobrandao@ yahoo.com. Autor para correspondência.

${ }^{3}$ Professor Doutor da Faculdade de Medicina Veterinária da Universidade Santo Amaro.

${ }^{4}$ Professor Titular do Departamento de Clíica Médica, FMVZ, USP.

${ }^{5}$ Médico Veterinário do HOVET, FMVZ, USP.

${ }^{6}$ Professor Doutor da Faculdade de Medicina, USP.
} 


\section{INTRODUÇÃO}

A liberação maciça de endotoxinas bacterianas na circulação sangüínea pode resultar na "Síndrome de Resposta Inflamatória Sistêmica", também conhecida como choque endotóxico, em que alterações hemodinâmicas decorrentes da vasodilatação e aumento da permeabilidade vascular periférica, desenvolvimento de hipotensão, redução marcante do fluido intravascular e perfusão sangüínea tecidual, isquemia e hipóxia de vários órgãos, conduzem freqüentemente à morte do animal (WEEREN \& MUIR, 1992). O fator desencadeante do choque endotoxêmico é um lipopolissacarídeo (LPS) de estrutura complexa, derivado da porção externa da membrana de bactérias Gram negativas, capaz de sensibilizar o organismo, induzindo à liberação sistêmica de citocinas, como o fator de necrose tumoral $(\mathrm{TNF}-\alpha)$ e as interleucinas tipo 1 e 6 (MIYAMOTO, 1993; CHANG et al.,1996).

Dentre as alterações metabólicas decorrentes do quadro de endotoxemia, as variações das concentrações séricas de glicose e a instalação progressiva de um quadro hipoglicêmico são reconhecidas como as principais responsáveis pela incapacidade de adaptação orgânica diante do quadro de endotoxemia (MILLER et al., 1980; BREITSCHWERDT $\boldsymbol{e t}$ al., 1981; LANG \& DOBRESCU, 1991).

A hiperglicemia é um achado freqüente durante a fase inicial do quadro endotoxêmico (WEIL et al., 1988; McGUINNESS et al., 1995; WITEK-JANUSEK \& YELICH, 1995; MUIR, 1998) e é decorrente do incremento da glicólise e da glicogenólise, promovido pela ação de catecolaminas e hormônios hiperglicemiantes (glucagon, hormônio do crescimento, etc). Os mecanismos regulatórios são capazes de manter provisoriamente a pressão sangüínea, a perfusão tecidual e a glicemia, compondo o que se denomina de fase hiperdinâmica do estado de choque, de melhor prognóstico. Com a progressão da doença e a incapacidade de manutenção do tono simpático por mais tempo, ocorre vasodilatação periférica e queda de pressão arterial, com conseqüente redução da perfusão sangüínea tecidual e da glicemia, contribuindo para a piora clínica progressiva, resultando na fase hipodinâmica do choque, considerada a fase final da doença (BREITSCHWERDT $\boldsymbol{e t}$ al., 1981; LANG \& DOBRESCU, 1991; MAITRA et al., 2000).

Considerando-se a hipoglicemia observada na fase hipodinâmica do choque endotóxico, de prognóstico grave, vários ensaios terapêuticos experimentais foram realizados na tentativa de restaurar a homeostasia glicêmica. As soluções de glicose acrescidas ou não com insulina e potássio, por via intravenosa, foram utilizadas por diversos autores, em cães com choque endotóxico (HINSHAW $\boldsymbol{e t}$ al., 1974; MANNY et al.,1978) no intuito de facilitar a entrada de glicose nas células, incrementando o metabolismo energético das mesmas.

Ao avaliarem as concentrações séricas de insulina nos quadros endotoxêmicos, CRYER et al. (1972), HINSHAW et al. (1974), MANNY et al.(1978), GROOWIN \& SCHAER (1989) e MUIR (1998) observaram sua redução, que foi creditada à ação de mecanismos de contra-regulação endógena entre insulina e glicose (hipoinsulinemia secundária à hipoglicemia), ou ainda como conseqüência da inibição da secreção insulínica pela ação de catecolaminas (epinefrina e norepinefrina) liberadas durante o processo endotoxêmico (CRYER et al., 1972; MANNY et al., 1978; BREITSCHWERDT et al., 1981).

Por outro lado, descreve-se também a hipersecreção insulínica nos quadros de endotoxemia (LEIFER $\boldsymbol{e t}$ al., 1985; BATTELINO et al., 1996; AGWUNOBI et al., 2000) e de acordo com YELICH \& FILKINS (1982) e VIRKAMAKI \& YKI-JARVINEN (1994) esta constitui-se em um dos achados mais graves e consistentes no quadro endotoxêmico, capaz de contribuir para o agravamento da hipoglicemia e piora do quadro clínico, resultando na morte do animal. De acordo com FELDMAN \& NELSON (1996), a avaliação conjunta das concentrações séricas absolutas de insulina e glicose, bem como das relações insulina/glicose e insulina/glicose corrigida, deve ser realizada com o objetivo de detectar a ocorrência de hipersecreção insulínica absoluta e/ou relativa.

Diante da controvérsia existente em relação às concentrações séricas de insulina em cães com endotoxemia e de estudos que mostrem o efeito benéfico da insulinoterapia, o presente estudo objetivou determinar as variações das concentrações séricas de glicose em cães com choque endotóxico e avaliar as concentrações séricas de insulina nos animais hipoglicêmicos, com a finalidade de se obterem subsídios para a instituição de terapia de reposição de insulina e glicose na fase hipodinâmica do choque.

\section{MATERIAIS E MÉTODOS}

Utilizaram-se vinte e cinco cães, machos e fêmeas, de raças variadas, com idade entre 2 e 6 meses, nos quais se constatou quadro de choque endotóxico decorrente da gastrenterite hemorrágica (diagnóstico presuntivo de parvovirose), provenientes do Serviço de Pronto Atendimento Médico do 
Departamento de Clínica Médica - Hospital Veterinário da Faculdade de Medicina Veterinária e Zootecnia da Universidade de São Paulo, no período compreendido entre Fevereiro de 1995 e Maio de 1996.

De cada animal, foram colhidos $5 \mathrm{~m} \ell$ de sangue total, por punção das veias jugular ou cefálica, os quais foram acondicionados em tubos sem anticoagulante ${ }^{\mathrm{a}}$ e centrifugados imediatamente para a obtenção do soro no qual foram avaliadas as concentrações de glicose e insulina. Os soros sangüíneos foram mantidos a $-20^{\circ} \mathrm{C}$ até seu processamento.

Para a determinação da glicemia utilizouse o método enzimático colorimétrico ${ }^{\mathrm{b}}$ em analisador automático ${ }^{c}$ do Laboratório de Análises Clínicas do Hospital Veterinário da Faculdade de Medicina Veterinária e Zootecnia da Universidade de São Paulo e para a determinação das concentrações séricas de insulina usou-se o método de radioimunoensaio em fase sólida ${ }^{\mathrm{d}}$, este realizado no Laboratório de Endocrinologia da Faculdade de Medicina da Universidade de São Paulo.

A obtenção da amostra de sangue de cada animal antecedeu a realização do tratamento medicamentoso, que consistiu de infusão intravenosa de solução cristalóide (Ringer com lactato de sódio) na dose de $90 \mathrm{~m} \ell / \mathrm{kg}$ na primeira hora e na sequência 10 a $12 \mathrm{~m} \ell / \mathrm{kg} / \mathrm{hora}$, acrescida de dextrose $50 \%$ na dose de 0,5 a $1,0 \mathrm{~m} \ell / \mathrm{kg} / \mathrm{hora}$, com o objetivo de manter as concentrações glicêmicas em torno de 100 a 200mg/dL, como recomendado por GROOWIN \& SCHAER (1989) e PURVIS \& KIRBY (1994).

\section{RESULTADOS E DISCUSSÃO}

Os animais foram divididos em três grupos, de acordo com os valores glicêmicos. Foram considerados hipoglicêmicos os animais em que se observou glicemia inferior a $80 \mathrm{mg} / \mathrm{dL}$ (13 animais); normoglicêmicos os animais com valores séricos de glicose entre 80 a $120 \mathrm{mg} / \mathrm{dL}$ (9 animais) e como hiperglicêmicos aqueles em que a glicemia foi superior a $120 \mathrm{mg} / \mathrm{dL}$ (3 animais), conforme figura 1 .

A ocorrência de hiperglicemia em 3 animais $(12 \%$ do total) pode ter sido decorrente de um incremento da glicogenólise promovida pela liberação de catecolaminas e hormônios hiperglicemiantes como resposta orgânica à sepse, na fase hiperdinâmica do choque endotóxico sendo, portanto, indicativo de prognóstico mais favorável, de acordo com CRYER et al. (1972), HARDIE (1995), McGUIINESS $\boldsymbol{e t}$ al. (1995) e WITEK-JANUSEK \& YELICH, 1995.

A hipoglicemia foi observada na maioria dos animais (52\% do total), coincidindo com as

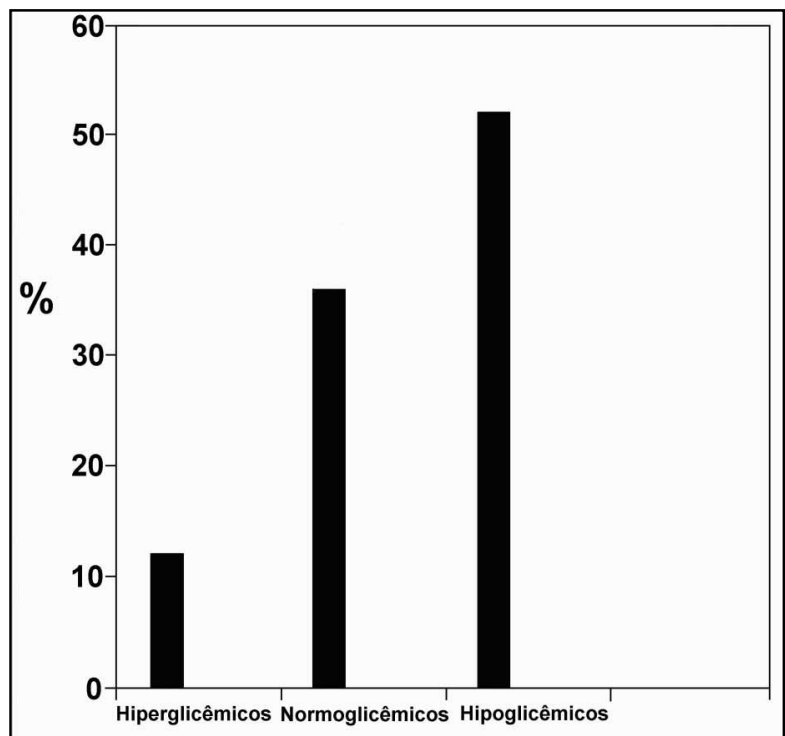

Figura 1 - Freqüência relativa de cães hiperglicêmicos (glicemia > $120 \mathrm{mg} / \mathrm{dL}$ ), normoglicêmicos (glicemia $80-120 \mathrm{mg} / \mathrm{dL}$ ) e hipoglicêmicos (glicemia $<80 \mathrm{mg} / \mathrm{dL}$ ), com choque séptico.

observações de HINSHAW et al. (1974), MANNY et al. (1978), BREITSCHWERDT et al. (1981), CALVERT \& GREENE (1986), LANG \& DOBRESCU (1991), BATTELINO et al. (1996) e MAITRA et al. (2000). A depleção dos estoques de glicogênio, bem como a maior utilização da via glicolítica anaeróbica para a manutenção de fonte energética-ATP, em função da hipoperfusão tecidual e da lesão mitocondrial pela ação direta das endotoxinas, nos casos de choque endotóxico, podem resultar na diminuição dos valores séricos de glicose (BREITSCHWERDT $\boldsymbol{e t} \boldsymbol{a} .$, 1981; LANG \& DOBRESCU, 1991).

Os valores séricos de insulina foram mensurados no grupo dos animais hipoglicêmicos com o intuito de se averiguar a existência de hiperinsulinemia absoluta ou relativa (Tabela 1 ). Consideraram-se normoinsulinêmicos os cães com concentração absoluta de insulina entre 5 e $20 \mu \mathrm{UI} / \mathrm{m} \ell$ de acordo com FELDMAN \& NELSON, (1996). A hiperinsulinemia relativa foi calculada pela análise das relações insulina/glicose e insulina/glicose corrigida (insulina ${ }_{x} 100 /$ concentração sérica de glicose 30). Os animais foram considerados hiperinsulinêmicos relativos quando os valores da relação insulina/glicose foram superiores a 0,235 e quando os valores da relação corrigida foram superiores a 30 , como portadores de hiperinsulinemia relativa corrigida, segundo FELDMAN $\boldsymbol{e t}$ al. (1986), LEIFER $\boldsymbol{e}$ t al. (1986), CAYWOOD et al. (1988) e FELDMAN \& NELSON (1996).

Ciência Rural, v. 31, n. 5, 2001. 
Tabela 1 - Valores de glicemia $(\mathrm{mg} / \mathrm{dl})$, insulina sérica $(\mu \mathrm{UI} / \mathrm{mL})$, relação insulina glicose e insulina glicose corrigida em cães hipoglicêmicos com choque séptico (glicemia $<80 \mathrm{mg} / \mathrm{dl})$.

\begin{tabular}{ccccc}
\hline $\begin{array}{c}\text { N. do } \\
\text { animal }\end{array}$ & $\begin{array}{c}\text { Glicose } \\
\text { sérica }\end{array}$ & $\begin{array}{c}\text { Insulina } \\
\text { sérica* }\end{array}$ & $\begin{array}{c}\text { Relação insuli- } \\
\text { na/glicose** }\end{array}$ & $\begin{array}{c}\text { Relação insuli- } \\
\text { na/glicose } \\
\text { corrigida*** }\end{array}$ \\
\hline 1 & 37,0 & 43,0 & 1,16 & 614,0 \\
2 & 35,0 & 5,3 & 0,15 & 106,0 \\
3 & 40,0 & $<5,0$ & 0,125 & 50,0 \\
5 & 55,0 & 37,0 & 0,67 & 148,0 \\
10 & 59,0 & 7,8 & 1,32 & 780,0 \\
11 & 63,0 & 20,0 & 0,31 & 60,6 \\
14 & 71,0 & 10,0 & 0,14 & 24,3 \\
15 & 63,0 & 3,1 & 0,049 & 9,39 \\
16 & 64,3 & 2,5 & 0,038 & 7,28 \\
17 & 75,0 & 5,0 & 0,06 & 11,11 \\
19 & 44,0 & 10,0 & 0,22 & 7,14 \\
24 & $(<) 26,0$ & 12,0 & 0,46 & 1200,0 \\
25 & 68,0 & 202,0 & 2,97 & 531,5 \\
\hline
\end{tabular}

* Valores de referência: aumentado $(>20 \mu \mathrm{UI} / \mathrm{mL})$ e diminuído $(<5 \mu \mathrm{UI} / \mathrm{mL})$. ** Valores de referência: aumentado $(>0,235)$.

*** Valores de referência: aumentado $(>30)$.

FELDMAN \& NELSON (1996).

A hiperinsulinemia absoluta foi observada nos animais 1,5 e 25 , nos quais as relações insulina/glicose e insulina/glicose corrigida também se encontravam aumentadas (Tabela 1). Os tumores pancreáticos de células $\beta$ devem estar dentre os diagnósticos diferenciais a serem excluídos na ocorrência de hiperinsulinemia absoluta ou relativa. Entretanto, pela dificuldade de diagnosticar esse tipo de afecção inferindo-se apenas sobre a análise dos valores séricos de glicose e insulina (KNOWLEN \& SCHALL, 1984; FELDMAN et al., 1986), deve-se utilizar o exame ultra-sonográfico de abdome para este fim. Devido ao histórico dos animais e o desaparecimento dos sinais clínicos após o tratamento, acredita-se que a hipersecreção insulínica tenha sido decorrente do quadro endotoxêmico e não de um provável tumor pancreático secretor de insulina (VIRKAMAKI \& YKI-JARVINEN, 1994; AGWUNOBI et al., 2000).

Embora nos animais de números 2, 3, $10,11,19$ e 24 não tenham sido observadas hiperinsulinemia absoluta (Tabela 1), quando se consideram os índices relativos de insulina/glicose e/ou insulina/glicose corrigida, estes animais apresentaram hiperinsulinemia relativa e/ou hiperinsulinemia relativa corrigida, podendo-se suspeitar da ocorrência de hiperinsulinismo ou resistência insulínica (FELDMAN \& NELSON, 1996; AGWUNOBI et al., 2000). Essa interpretação deve ser realizada cautelosamente, segundo LEIFER et al. (1985) já que, por se tratar de um índice relativo, pode haver a ocorrência de resul- tados falso-positivos, como na avaliação de animais hipoglicêmicos portadores de hepatopatias ou septicemias (BREITSCHWERDT $\boldsymbol{e}$ t al., 1981; KNOWLEN \& SCHALL, 1984; FELDMAN et al., 1986; LEIFER et al., 1986).

Apenas nos animais 14, 15, 16 e 17 não foram observadas alterações nos valores séricos absolutos ou relativos de insulina, o que permite concluir que, no momento da avaliação não havia nenhum indicativo de hipoglicemia associada à hiperinsulinemia. Ao contrário, nos animais de número 15 e 16, além da hipoglicemia observou-se hipoinsulinemia absoluta, indicando um possível esgotamento das células $\beta$ pancreáticas ou um equilíbrio na produção de insulina na presença de hipoglicemia, uma vez que as concentrações séricas desse hormônio devem acompanhar as de seu substrato metabólico, mantendo o equilíbrio homeostático e a manutenção da normoglicemia (FELDMAN \& NELSON, 1996).

A hiperinsulinemia absoluta ou relativa, também relatada por diversos autores (YELICH \& FILKINS, 1982; LEIFER et al., 1985; BATTELINO et al., 1996) pode ser creditada à ocorrência de hipersecreção insulínica (YELICH \& FILKINS, 1982) que em parte pode estar contribuindo para o agravamento da hipoglicemia. Ainda, de acordo com HOTAMISLIGIL $\boldsymbol{e t}$ al. (1994) e HOTAMISLIGIL \& SPIEGELMAN (1994), pode ser uma conseqüência do desenvolvimento de resistência periférica à insulina promovida por hormônios contraregulatórios hiperglicemiantes (catecolaminas, glucagon, cortisol e GH-hormônio de crescimento) ou também pela ação do TNF- $\alpha$ (fator de necrose tumoral) que pode interferir, de modo a incrementar o desenvolvimento da resistência periférica à insulina (VIRKAMAKI \& YKI-JARVINEN, 1994; AGWUNOBI et al., 2000).

A constatação de que mais da metade dos animais hipoglicêmicos, na fase mais avançada do choque, apresentaram hiperinsulinemia absoluta ou relativa revelou a necessidade de uma avaliação cautelosa do paciente antes de se proceder à indicação terapêutica de insulina, a qual se restringirá àqueles em que houver resistência periférica à sua ação, ou ainda, naqueles casos em que a hipoglicemia for acompanhada de hipoinsulinemia, sendo nesse caso mandatória a associação de insulina e glicose (MANNY et al.,1978) .

\section{AGRADECIMENTOS}

Ao $\mathrm{CNPq}$ pelo aporte financeiro e a todos os professores, pós-graduandos, médicos veterinários contratados, residentes, alunos e funcionários do HOVET/USP que contribuíram para a realização deste estudo. 


\section{FONTES DE AQUISIÇÃO}

a -Tubos Vacutainer ${ }^{\circledR}$ SST ${ }^{\circledR}$ Gel and clot activator. Becton e Dickinson Indústria Cirúrgica Ltda. Curitiba, PR.

b - Kit glicose Merck System. Merck S.A. Indústrias Químicas. São Paulo, SP.

c - Analisador automático Technicon RA-100. Bayer S.A. São Paulo, SP.

d - Kit DPC (Distribution Products Corporation) - Insulina RIA. Estados Unidos da América.

\section{REFERÊNCIAS BIBLIOGRÁFICAS}

AGWUNOBI, A.O., REID, C., MAYCOCK, P., et al. Insulin resistance and substrate utilization in human endotoxemia. Journal of Clinical Endocrinology and Metabolism, v.85, n.10, p.3770-3778, 2000 .

BATtElinO, T., GOTO, M., KRZISNIK, C., et al. Tissue glucose transport in suckling rats with endotoxic shock. Shock, v.6, n.4, p.259-262, 1996.

BREITSCHWERDT, E.B., LOAR, A.S., HRIBERNIK, T.N., et al. Hypoglycemia in four dogs with sepsis. Journal of American Veterinary Medical Association, v.178, n.10, p.1072-1076, 1981.

CALVERT, C.A., GREENE, C.E. Bacteremia in dogs: Diagnosis, treatment and prognosis. The Compendium on Continuing Education, v.8, n.3, p.179-186, 1986.

CAYWOOD, D.D., KLAUSNER, J.S., O'LEARY, T.P., et al. Pancreatic insulin-secreting neoplasms: clinical, diagnostic, and prognostic features in 73 dogs. Journal of the American Animal Hospital Association, v.24, september/october, p.577584, 1988.

CHANG, C.K., GATAN, M., SCHUMER, W. Efficacy of antitumor necrosis factor polyclonal antibody on phosphoenolpyruvate carboxykinase expression in septic and endotoxemic rats. Shock, v.6, n.1, p.57-60, 1996.

CRYER, P.E.; CORAN, A.G., SODE, J., et al. Lethal Escherichia coli septicemia in the baboon: alpha-adrenergic inhibition of insulin secretion and it's relationship to the duration of survival. Journal of Laboratory and Clinical Medicine, v.79, p.622-638, 1972.

FELDMAN, E.C., NELSON, R.W. Canine and feline endocrinology and reproduction. 2.ed. Philadelphia : Saunders, 1996. Cap.12: Beta-cell neoplasia: insulinoma: p.422-441.

FELDMAN, E.C., SCHALL, W.D., KRUTH, S.A., et al. Amended insulin: glucose ratio. Journal of American Veterinary Medical Association, v.188, n.11, p.1227-1230, 1986.

GROOWIN, J.K., SCHAER, M. Septic Shock. Veterinary Clinics of North America: Small Animal Practice, v.19, n.6, p.1239-1258, 1989.

HARDIE, E.M. Life-threatening in bacterial infections. The Compendium on Continuing Education, v.17, n.6, p.763777, 1995.

HINSHAW, L.B., PEYTON, M.D., ARCHER, L.T., et al. Prevention of death in endotoxin shock by glucose administration. Surgery, Gynecology \& Obstetrics, v.139, p.851-858, 1974.

HOTAMISLIGIL, G.S., SPIEGELMAN, B.M. Perspectives in diabetes. Tumor necrosis factor $\alpha$ : a key component of the obesity - diabetes link. Diabetes, v.43, p.1271-1278, 1994.
HOTAMISLIGIL, G.S, MURRAY, D.L., CHOY, L.N., et al. Tumor necrosis factor $\alpha$ inhibits signaling from the insulin receptor. Proceedings of the Nacional Academy of Sciences of the United States of America, v.91, p.4854-4858, 1994.

KNOWLEN, G.G., SCHALL, W.D. The amended insulinglucose ratio. Is it really better? Journal of American Veterinary Medical Association, v.185, p.397-399, 1984.

LANG, C.H., DOBRESCU, C. Sepsis-induced increases in glucose uptake by macrophage-rich tissues persist during hypoglycemia. Metabolism, v.40, n.6, p.585-593, 1991.

LEIFER, C.E., PETERSON M.E., MATUS, R.E., $\boldsymbol{e}$ t al. Hypoglycemia associated with nonislet cell tumor in 13 dogs. Journal of American Veterinary Medical Association, v.186, p.53-55, 1985 .

LEIFER, C.E., PETERSON M.E., MATUS, R.E. Insulinsecreting tumor: Diagnosis and medical and surgical management in 55 dogs. Journal of American Veterinary Medical Association, v.188, n. 1, p.60-64, 1986.

MAITRA, S.R., WOJNAR, M.M., LANG, C.H. Alterations in tissue glucose uptake during the hyperglycemic and hypoglycemic phases of sepsis. Shock, v.13, n.5, p.379-385, 2000.

MANNY, J., RABINOVICI, N., SCHILER, M. Effect of glucoseinsulin-potassion on survival in experimental endotoxic shock. Surgery, Gynecology \& Obstetrics, v.147, p.405-409, 1978.

McGUINNESS, O.P., LACY, D.B., ANDERSON, J Effect of acute glucagon removal on metabolic response to infection in conscious dog. American Journal of Physiology, v.268, n.1, p.92-99, 1995.

MILLER, S.I.; WALLACE, R.J., MUSHER, D.M., et al. Hipoglicemia as a manifestation of sepsis. The American Journal of Medicine, v.68, p.649-654, 1980.

MIYAMOTO, T. Changes of citokine activities and other parameters in experimentally induced endotoxin shock in dogs. Japanese Journal of Veterinary Research, v.41, n.1, p.32, 1993.

MUIR, W.W. Shock. The Compendium on Continuing Education, v.20, n.5, p.549-566, 1998.

PURVIS, D., KIRBY, R. Systemic inflammatory response syndrome - Septic Shock. Veterinary Clinics of North America: Small Animal Practice, v.24, n.6, p.1225-1247, 1994.

VIRKAMAKI, A., YKI-JARVINEN, H Mechanisms of insulin resistance during acute endotoxemia. Endocrinology, v.134, n.5, p.2072-2078, 1994.

WEEREN, F.R., MUIR, W.W. Clinical aspects of septic shock and compreensive approaches to treatment in dogs and cats. Journal of American Veterinary Medical Association, v.200, n.12, p.1859-1870, 1992.

WEIL, M.H., VON PLANTA, M., RACKOW, E.C. Acute circulatory failure (shock). In: BRAUNWALD, E. A Textbook of cardiovascular medicine. 3ed Philadelphia : Saunders, 1988. p.561-577.

WITEK-JANUSEK, L., YELICH, M.R. Role of the cortex and medulla in the young rat's glucoregulatory response to endotoxin. Shock, v.3, n.6, p.434-439, 1995.

YELICH, M.R., FILKINS, J.P. Insulin hypersecretion and potentation of endotoxin shock in the rat Circulatory Shock, v.9, p.589-603, 1982. 